\title{
Synthesis and biological screening of some novel pyrazolo[3',4':4,5]thieno[2,3-d]pyrimidin-8-ones via a Gewald reaction
}

\author{
Shailesh J. Vaghasiya, Dipti K. Dodiya, Amit R. Trivedi, and Viresh H. Shah* \\ Chemical Research Laboratory, Department of chemistry, \\ Saurashtra University, Rajkot-360 005, India \\ E-mail: Shah_v_h@yahoo.com
}

\begin{abstract}
A new synthetic route is proposed for the synthesis of 3,6-dimethyl-6-aryl-1,5,6,7-tetrahydro$8 H$-pyrazolo[3',4':4,5] thieno[2,3-d]pyrimidin-8-ones 3a-h from 5-amino-3-methyl-1Hthieno[3,2-c]pyrazole-6-carbonitrile 2. Synthesis of the key compound 2 was accomplished via a Gewald reaction. The newly synthesized compounds $\mathbf{3 a - h}$ were characterized by elemental analysis, IR, ${ }^{1} \mathrm{H}-\mathrm{NMR},{ }^{13} \mathrm{C}-\mathrm{NMR}$ and mass spectroscopic investigation. All the compounds 3a-h were screened for their antitubercular activity against Mycobacterium tuberculosis $H_{37} R V$.
\end{abstract}

Keywords: Pyrazolo[3',4':4,5]thieno[2,3-d]pyrimidin-8-ones, carbonitrile, Gewald reaction, antitubercular activity, antimycobacterial activity

\section{Introduction}

Pyrazole containing compounds have practical applications in the medicinal and agrochemical field and the biological activity of pyrazoles ${ }^{1,2}$ and its derivatives are well documented. The pyrazole ring has shown to be the basic moiety for a number of dyes and drugs ${ }^{3,4}$. Substituted pyrazolopyrimidinones are found to be useful as cardiotonic, ${ }^{5}$ herbicidal ${ }^{6}$ and antiviral ${ }^{7}$ agents. $^{2}$ Literature survey reveals that substituted pyrazolopyrimidinones are potent and selective inhibitors of type 5 cyclic guanosine-3', 5' -monophosphate phosphodiesterase (cGMP) PDE-5 ${ }^{8,9}$ and, as such, have utility in the treatment of male erectile dysfunction (MED) and female sexual dysfunction $(\mathrm{FSD})^{10}$. C-6 substituted pyrimidinone and pyrimidindione derivatives have shown selective antitumor, ${ }^{11}$ antiviral, ${ }^{12}$ antitubercular ${ }^{13}$ and antifungal activity ${ }^{14}$. The above mentioned references suggest the importance of testing this family of compounds as broad-spectrum drugs.

In search of bioactive molecules and in continuation of our previous work $^{15-18}$ in developing syntheses of polyfunctionally substituted heterocyclic compounds, we report a novel synthetic approach for the synthesis of 3,6-dimethyl-6-aryl-1,5,6,7-tetrahydro- $8 H$ - 
pyrazolo[3',4':4,5]thieno[2,3-d]pyrimidin-8-ones 3a-h from key compound 5-amino-3-methyl$1 H$-thieno[3,2-c]pyrazole-6-carbonitrile 2, which was synthesized via a Gewald ${ }^{19-24}$ reaction in the presence of sulfur and morpholine. All the newly synthesized compounds 3a-h were evaluated for in vitro antimycobactrial activity against Mycobacterium tuberculosis $H_{37} R V$.

\section{Results and Discussion}

Synthesis of 5-amino-3-methyl-1H-thieno[3,2-c]pyrazole-6-carbonitrile 2 was accomplished by refluxing (5-methyl-2,4-dihydro-3H-pyrazol-3-ylidene)malononitrile $\mathbf{1}$ and sulfur in the presence of morpholine for $6 \mathrm{hrs}$. The IR spectrum of 2 revealed the appearance of bands characteristics of stretching vibrations of 3420-3305 $\mathrm{cm}^{-1}\left(-\mathrm{NH}_{2}\right), 2234 \mathrm{~cm}^{-1}(-\mathrm{CN}), 1645 \mathrm{~cm}^{-1}(\mathrm{C}=\mathrm{N})$ groups.<smiles>CC1=NNC(=O)C1</smiles>

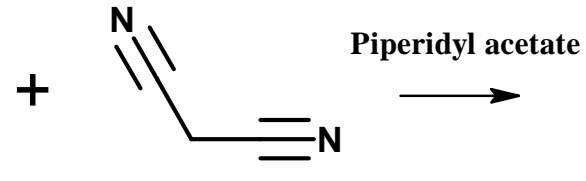

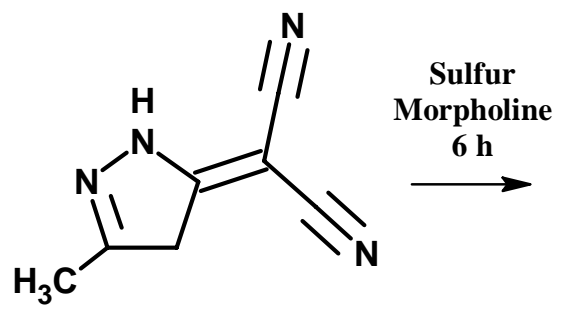

$1(52 \%)$<smiles>Cc1n[nH]c2c(C#N)c(N)sc12</smiles>

$2(58 \%)$

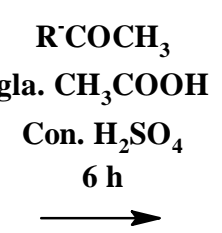

gla. $\mathrm{CH}_{3} \mathrm{COOH}$
Con. $\mathrm{H}_{2} \mathrm{SO}_{4}$
$\underset{6 \mathrm{~h}}{\longrightarrow}$<smiles>[R]C1(C)NC(=O)c2c(sc3c(C)n[nH]c23)N1</smiles>

3a-h (42-56\%)

\section{R= Phenyl, \\ 2-hydroxy phenyl, 4-fluoro phenyl 4-chloro phenyl, 4-bromo phenyl 4-nitro phenyl, 4-methyl phenyl, 4-methoxy phenyl.}

\section{Scheme-1}


Furthermore, the ${ }^{1} \mathrm{H}$ NMR spectra of compound 2 showed signals at $\delta 2.68$ as a singlet for ($\left.\mathrm{CH}_{3}\right), \delta 4.0$ as a singlet for $\left(-\mathrm{NH}_{2}\right), \delta 13.68$ as a singlet for the $(-\mathrm{NH})$ group, which confirmed the structure. Compound 2 on reaction with different aromatic ketones in glacial acetic acid furnished the title compounds 6-aryl-3,6-dimethyl-1,5,6,7-tetrahydro- $8 \mathrm{H}$-pyrazolo $\left[3^{\prime}, 4^{\prime}: 4,5\right]$ thieno[2,3-d] pyrimidin-8-ones 3a-h in excellent yields.

The IR spectroscopic investigation of 3a-h revealed characteristic bands in the range of $2975-2968 \mathrm{~cm}^{-1}\left(-\mathrm{CH}_{3}\right), 1687-1678 \mathrm{~cm}^{-1}(\mathrm{C}=\mathrm{O}), 690-680 \mathrm{~cm}^{-1}(\mathrm{C}-\mathrm{S}-\mathrm{C})$ etc. Formation of compounds 3a-h from 2 was confirmed by ${ }^{1} \mathrm{H}$ NMR signals that appeared as a singlet around $\delta$ 8.0 for protons of $-\mathrm{NH}$ (pyrimidine nucleus) and multiplet in the range of $\delta 7.0-8.0$ (aromatic protons). Further, ${ }^{13} \mathrm{C}$ NMR spectra exhibited confirmatory signals of the carbonyl carbon and the methyl carbon around $\delta 164$ and $\delta 28$ respectively.

\section{Biological activity}

The in vitro antimycobacterial activity was assayed by the Tuberculosis Antimicrobial Acquisition Coordinating Facility (TAACF) antituberculosis drug discovery program, coordinated by the Southern Research Institute (Birmingham, Ala.) under the direction of the National Institute of Allergy and Infectious Diseases, U.S.A. All the compounds (3a-h) were initially screened against Mycobacterium tuberculosis $H_{37} R V$ (ATCC 27294) (American Type Culture Collection, Manassas, Va.) at the single concentration of $6.25 \mu \mathrm{g} / \mathrm{ml}$ in BACTEC 12B medium using a broth micro dilution assay, the microplate Alamar blue assay (MABA) ${ }^{25}$. Compounds exhibiting fluorescence were tested in the BACTEC 460 radiometric system ${ }^{26}$. Compounds demonstrating at least $90 \%$ inhibition in the primary screening were retested at lower concentrations by serial dilution against Mycobacterium tuberculosis $H_{37} R V$ to determine the actual MIC, using the micro plate alamar blue assay (MABA) method.

Concurrent with the determination of MICs, compounds were tested for cytotoxicity (50\% inhibitory concentration $\left[\mathrm{IC}_{50}\right]$ ) in Vero cell line to determine the selectivity index (SI), defined as the ratio of the measured IC50 in VERO cells to the MIC values. Compound 3d showed a MIC value of $3.13 \mu \mathrm{g} / \mathrm{ml}$, IC50 value of 1.660 and SI of 0.5322 . The results of antitubercular activities are represented in Table 1. 
Table 1. Antitubercular activity screening data of the synthesized compounds

\begin{tabular}{|c|c|c|c|c|c|c|c|}
\hline $\begin{array}{l}\text { Sr. } \\
\text { No. }\end{array}$ & $\mathrm{R}$ & $\begin{array}{c}\text { Molecular } \\
\text { Formula } \\
\end{array}$ & Assay & $\begin{array}{l}\text { Drug } \\
\text { Units }\end{array}$ & $\mathrm{IC}_{50}$ & $\mathrm{IC}_{90}$ & Activity \\
\hline 3a & Phenyl & $\mathrm{C}_{15} \mathrm{H}_{14} \mathrm{~N}_{4} \mathrm{OS}$ & MABA & $\mu \mathrm{g} / \mathrm{mL}$ & $>100$ & $>100$ & Inactive \\
\hline $3 \mathbf{b}$ & 4-Hydroxyphenyl & $\mathrm{C}_{15} \mathrm{H}_{14} \mathrm{~N}_{4} \mathrm{O}_{2} \mathrm{~S}$ & MABA & $\mu \mathrm{g} / \mathrm{mL}$ & $>100$ & $>100$ & Inactive \\
\hline $3 c$ & 4-Fluorophenyl & $\mathrm{C}_{15} \mathrm{H}_{14} \mathrm{~N}_{4} \mathrm{OSF}$ & MABA & $\mu \mathrm{g} / \mathrm{mL}$ & $>100$ & $>100$ & Inactive \\
\hline 3d & 4-Chlorophenyl & $\mathrm{C}_{15} \mathrm{H}_{14} \mathrm{~N}_{4} \mathrm{OSCl}$ & MABA & $\mu \mathrm{g} / \mathrm{mL}$ & 1.660 & 1.336 & Active \\
\hline $3 \mathbf{e}$ & 4-Bromophenyl & $\mathrm{C}_{15} \mathrm{H}_{14} \mathrm{~N}_{4} \mathrm{OSBr}$ & MABA & $\mu \mathrm{g} / \mathrm{mL}$ & $>100$ & $>100$ & Inactive \\
\hline $3 f$ & 4-nitrophenyl & $\mathrm{C}_{15} \mathrm{H}_{13} \mathrm{~N}_{5} \mathrm{O}_{3} \mathrm{~S}$ & MABA & $\mu \mathrm{g} / \mathrm{mL}$ & $>100$ & 21.30 & Weakly Active \\
\hline $3 g$ & 4-Methylphenyl & $\mathrm{C}_{16} \mathrm{H}_{16} \mathrm{~N}_{4} \mathrm{OS}$ & MABA & $\mu \mathrm{g} / \mathrm{mL}$ & $>100$ & $>100$ & Inactive \\
\hline $3 \mathbf{h}$ & 4-Methoxyphenyl & $\mathrm{C}_{16} \mathrm{H}_{16} \mathrm{~N}_{4} \mathrm{O}_{2} \mathrm{~S}$ & MABA & $\mu \mathrm{g} / \mathrm{mL}$ & $>100$ & $>100$ & Inactive \\
\hline
\end{tabular}

The present study on the synthesis and biological screening of some novel pyrazolo[3', $\left.4^{\prime}: 4,5\right]$ thieno[2,3-d]pyrimidin-8-ones has established the discovery of new types of pyrazolopyrimidinones analogues with significant and promising anti-tuberculosis activity against Mycobacterium tuberculosis $H_{37} R V$. Biological studies clearly indicate that the presence of the 4-chloro phenyl substituent in the 2 position of the pyrimidine ring of the pyrazolo [3',4':4,5] thieno[2,3-d]pyrimidin-8-ones gives useful biological activity. This effective derivative is ideally suited for further modifications to obtain more efficacious antimycobacterial compounds.

\section{Experimental Section}

General Procedures. Melting points were determined in open capillaries and are uncorrected. The IR spectra were recorded on Nicolet Impact 410 FT IR spectrophotometer using KBr pellets. ${ }^{1} \mathrm{H}$ and ${ }^{13} \mathrm{C}$ NMR spectra were recorded on Bruker 300-MHz FT NMR spectrometer in $\mathrm{CDCl}_{3}$ and DMSO-d6 with TMS as an internal standard. Mass spectra were recorded on ThermoFinnigan-MAT, Bremen (Model MAT8200) spectrometer and elemental analysis was carried out using Heraus CHN rapid analyzer. All chemicals were purchased from Aldrich Chemical Company (USA) and were used as received unless otherwise noted. Solvents used for the chemical synthesis were of laboratory and analytical grade, and were used without further purification.

Preparation of (5-methyl-2,4-dihydro-3H-pyrazol-3-ylidene)malononitrile (1). A mixture of 5-methyl-2,4-dihydro-3H-pyrazol-3-one $(0.98 \mathrm{~g}, 0.01 \mathrm{~mol})$ and malononitrile $(66 \mathrm{~mL}, 0.01 \mathrm{~mol})$ was heated under reflux condition for 6 hours in the presence of piperidyl acetate in a catalytic amount. The reaction mixture was poured into ice cold water; the crude product was filtered, dried and recrystallized from $95 \%$ ethanol. Yield 52\%, mp. 190-192 ${ }^{\circ} \mathrm{C}$. IR (KBr): 3226, 2210, $1658 \mathrm{~cm}^{-1} .{ }^{1} \mathrm{H}$ NMR $\left(300 \mathrm{MHz}, \mathrm{CDCl}_{3}\right): \delta 2.21\left(\mathrm{~s}, 3 \mathrm{H},-\mathrm{CH}_{3}\right), 1.91\left(\mathrm{~s}, 2 \mathrm{H},-\mathrm{CH}_{2}\right), 7.13(\mathrm{~s}, 1 \mathrm{H},-$ 
$\mathrm{NH}) ;{ }^{13} \mathrm{C}-\mathrm{NMR}\left(75 \mathrm{MHz}, \mathrm{DMSO}-d_{6}\right): \delta 19.5,35.1,61.8,113.9,153.5,181.3$. Mass $(\mathrm{m} / \mathrm{z}): 146$. Anal. (\%) for $\mathrm{C}_{7} \mathrm{H}_{6} \mathrm{~N}_{4}$, Calcd. C, 57.53; H, 4.14; N, 38.34. Found: C, 57.31; H, 4.02; N, 38.16 .

Preparation of 5-amino-3-methyl-1H-thieno[3,2-c]pyrazole-6-carbonitrile (2). A mixture of (5-methyl-2,4-dihydro-3H-pyrazol-3-ylidene)malononitrile 1 (1.46 g, $0.01 \mathrm{~mol})$ and sulfur (0.32 $\mathrm{g}, 0.01 \mathrm{~mol})$ was heated under reflux condition in the presence of morpholine $(0.87 \mathrm{~mL})$ for 6 hours. The reaction mixture was poured into ice cold water; the crude product was filtered, dried and recrystallized from $95 \%$ ethanol. Yield 58\%, mp. 206-208 ${ }^{\circ} \mathrm{C}$. IR (KBr): $3420-3305,2234$, $1645 \mathrm{~cm}^{-1} .{ }^{1} \mathrm{H}$ NMR $\left(300 \mathrm{MHz} \mathrm{CDCl}_{3}\right): \delta 2.689\left(\mathrm{~s}, 3 \mathrm{H},-\mathrm{CH}_{3}\right), 4.0\left(\mathrm{~s}, 2 \mathrm{H},-\mathrm{NH}_{2}\right), 13.72(\mathrm{~s}, 1 \mathrm{H},-$ $\mathrm{NH}) ;{ }^{13} \mathrm{C}-\mathrm{NMR}\left(75 \mathrm{MHz}, \mathrm{DMSO}-d_{6}\right): \delta 14.6,105.8,110.8,114,135,136.5,144$. Mass $(\mathrm{m} / \mathrm{z})$ : 178. Anal. (\%) for $\mathrm{C}_{7} \mathrm{H}_{6} \mathrm{~N}_{4} \mathrm{~S}$, Calcd. C, 47.18, H, 3.39, N, 31.44, S, 17.99. Found: C, 46.95, H, $3.19 ; \mathrm{N}, 31.23 ; \mathrm{S}, 17.75$.

General procedure for synthesis of 3,6-dimehtyl-6-aryl-1,5,6,7-tetrahydro-8Hpyrazolo[3',4':4,5]thieno[2,3-d]pyrimidin-8-ones (3a-h)

A mixture of 5-amino-3-methyl- $1 H$-thieno[3,2-c]pyrazole-6-carbonitrile 2 (1.98 g, $0.01 \mathrm{~mol})$ and an appropriate ketone $(0.01 \mathrm{~mol})$ in glacial acetic acid in presence of con. sulfuric acid was heated under reflux condition for 6 hours. The reaction mixture was poured into ice cold water; the crude product was filtered, dried and recrystallized from $95 \%$ ethanol.

6-(Phenyl)-3,6-dimethyl-1,5,6,7-tetrahydro-8H-pyrazolo[3',4':4,5]thieno[2,3-d]pyrimidin-8one (3a). Yield 45\%, mp. 210-212 ${ }^{\circ} \mathrm{C}$; IR (KBr): 3327, 3105, 2970, 1680, $681 \mathrm{~cm}^{-1} .{ }^{1} \mathrm{H}$ NMR $\left(300 \mathrm{MHz}, \mathrm{CDCl}_{3}\right): \delta 2.73\left(\mathrm{~s}, 3 \mathrm{H},-\mathrm{CH}_{3}\right.$, pyrazole), 2.12 (s, 3H, $-\mathrm{CH}_{3}$, pyrimidine), $8.01(\mathrm{~s}, 1 \mathrm{H}$, $\mathrm{NH}$, pyrimidine), 7.22-7.04 (m, 4H, Ar-H), 13.75 (s, 1H, -NH, pyrazole); ${ }^{13} \mathrm{C}-\mathrm{NMR}(75 \mathrm{MHz}$, DMSO-d $\left.{ }_{6}\right): \delta 14.4,28.4,103.1,126.4,126.8,128.6,135,141.9,142.8,144.0,161.9,164.2$. Mass $(\mathrm{m} / \mathrm{z})$ : 298. Anal. (\%) for $\mathrm{C}_{15} \mathrm{H}_{14} \mathrm{~N}_{4} \mathrm{OS}$, Calcd. C, 60.38; H, 4.73; N, 18.78; S, 10.75. Found: C, $60.19 ; \mathrm{H}, 4.56 ; \mathrm{N}, 18.31 ; \mathrm{S}, 10.69$.

6-(4-Hydroxyphenyl)-3,6-dimethyl-1,5,6,7-tetrahydro-8H-pyrazolo[3',4':4,5]thieno[2,3-d] pyrimidin-8-one (3b). Yield 44\%, mp. 191-192 ${ }^{\circ} \mathrm{C}$. IR (KBr): 3490-3100, 3120, 2974, 1682 , $684 \mathrm{~cm}^{-1} .{ }^{1} \mathrm{H}$ NMR $\left(300 \mathrm{MHz}, \mathrm{CDCl}_{3}\right): \delta 2.17\left(\mathrm{~s}, 3 \mathrm{H},-\mathrm{CH}_{3}\right.$, pyrimidine), $2.70\left(\mathrm{~s}, 3 \mathrm{H},-\mathrm{CH}_{3}\right.$, pyrazole), 4.52 (s, 1H, -OH), 6.73-7.04 (m, 4H, Ar-H), 8.09 (s, 1H, -NH, pyrimidine), 13.75 (s, $1 \mathrm{H},-\mathrm{NH}$, pyrazole); ${ }^{13} \mathrm{C}-\mathrm{NMR}\left(75 \mathrm{MHz}, \mathrm{DMSO}-d_{6}\right): 14.4,28.2,66.1,105,115.1,124.7,128.7$, 135.1, 141.8, 144.8, 156, 162.3, 164.6. Mass (m/z): 314. Anal. (\%) for $\mathrm{C}_{15} \mathrm{H}_{14} \mathrm{~N}_{4} \mathrm{O}_{2} \mathrm{~S}$, Calcd. C, 57.31; H, 4.49; N, 17.82; S, 10.20. Found: C, 57.03; H, 4.25; N, 17.68; S, 10.13.

\section{6-(4-Fluorophenyl)-3,6-dimethyl-1,5,6,7-tetrahydro-8H-pyrazolo[3',4':4,5]thieno[2,3-d]}

pyrimidin-8-one (3c). Yield 42\%, mp. 190-192 ${ }^{\circ} \mathrm{C}$. IR (KBr): 3335, 3120, 2975, 1685, 689, 710 $\mathrm{cm}^{-1} .{ }^{1} \mathrm{HNMR}\left(300 \mathrm{MHz}, \mathrm{CDCl}_{3}\right): \delta 2.02\left(\mathrm{~s}, 3 \mathrm{H},-\mathrm{CH}_{3}\right.$, pyrimidine), 2.75 (s, 3H, - $\mathrm{CH}_{3}$, pyrazole), 6.91-7.21 (m, 4H, Ar-H), 8.07 (s, 1H, -NH, pyrimidine), 13.73 (s, 1H, -NH, pyrazole); ${ }^{13} \mathrm{C}-\mathrm{NMR}$ (75 MHz, DMSO- $\left.d_{6}\right): \delta 14.1,29,66.9,105.8,114,128.9,135,137.9,141.5,144.9,159,161.9$, 165. Mass (m/z): 316. Anal. (\%) for $\mathrm{C}_{15} \mathrm{H}_{14} \mathrm{~N}_{4} \mathrm{OSF}$, Calcd. C, 56.95; H, 4.14; N, 17.72; S, 10.14. Found: C, 56.60; H, 4.01; N, 17.51; S, 10.01. 
6-(4-Chlorophenyl)-3,6-dimethyl-1,5,6,7-tetrahydro-8H-pyrazolo[3',4':4,5]thieno[2,3-d] pyrimidin-8-one (3d). Yield 42\%, mp. 224-226 ${ }^{\circ} \mathrm{C}$. IR (KBr): 3340, 3115, 2975, 1683, 685, 750 $\mathrm{cm}^{-1} .{ }^{1} \mathrm{H}$ NMR $\left(300 \mathrm{MHz}, \mathrm{CDCl}_{3}\right): \delta 2.08\left(\mathrm{~s}, 3 \mathrm{H},-\mathrm{CH}_{3}\right.$, pyrimidine), $2.73\left(\mathrm{~s}, 3 \mathrm{H},-\mathrm{CH}_{3}\right.$, pyrazole), 7.03-7.21 (m, 4H, Ar-H), 8.02 (s, 1H, -NH, pyrimidine), 13.72 (s, 1H, -NH, pyrazole); ${ }^{13} \mathrm{C}-\mathrm{NMR}\left(75 \mathrm{MHz}, \mathrm{DMSO}-d_{6}\right): \delta 28.8,104,127,128.2,131.9,136.5,140,141,144.9,161.9$, 165. Mass $(\mathrm{m} / \mathrm{z}): 332.55$. Anal. (\%) for $\mathrm{C}_{15} \mathrm{H}_{14} \mathrm{~N}_{4} \mathrm{OSCl}$, Calcd. C, 54.13; H, 3.94; N, 16.83; S, 9.63. Found: C, 54.01; H, 3.72; N, 16.56; S, 9.41.

6-(4-Bromophenyl)-3,6-dimethyl-1,5,6,7-tetrahydro-8H-pyrazolo[3',4':4,5]thieno[2,3-d] pyrimidin-8-one (3e). Yield 45\%, mp. 240-243 ${ }^{\circ} \mathrm{C}$. IR (KBr): 3337, 3110, 2972, 1683, 687, 770 $\mathrm{cm}^{-1} .{ }^{1} \mathrm{H}$ NMR $\left(300 \mathrm{MHz}, \mathrm{CDCl}_{3}\right): \delta 2.04\left(\mathrm{~s}, 3 \mathrm{H},-\mathrm{CH}_{3}\right.$, pyrimidine), $2.76\left(\mathrm{~s}, 3 \mathrm{H},-\mathrm{CH}_{3}\right.$, pyrazole), 7.03-7.36 (m, 4H, Ar-H), 8.05 (s, 1H, -NH, pyrimidine), 13.77 (s, 1H, -NH, pyrazole); ${ }^{13} \mathrm{C}-\mathrm{NMR}\left(75 \mathrm{MHz}, \mathrm{DMSO}-d_{6}\right): \delta 14.4,29.1,66.5,106.1,121,128.6,135,141.8,145.1,161.2$, 166. Mass (m/z): 377. Anal. (\%) for $\mathrm{C}_{15} \mathrm{H}_{14} \mathrm{~N}_{4} \mathrm{OSBr}$, Calcd. C, 47.76; H, 3.47; N, 14.85; S, 8.50. Found: C, 47.43, H, 3.26; N, 14.56; S, 8.3.

6-(4-Nitrophenyl)-3,6-dimethyl-1,5,6,7-tetrahydro-8H-pyrazolo[3',4':4,5]thieno[2,3-d] pyrimidin-8-one (3f). Yield 42\%, mp. $257-259{ }^{\circ} \mathrm{C}$. IR (KBr): 3340, 3114, 2969, 1682, $688 \mathrm{~cm}^{-1}$. ${ }^{1} \mathrm{H}$ NMR (300 MHz, $\left.\mathrm{CDCl}_{3}\right): \delta 2.04\left(\mathrm{~s}, 3 \mathrm{H},-\mathrm{CH}_{3}\right.$, pyrimidine), 2.76 (s, 3H, $-\mathrm{CH}_{3}$, pyrazole), 7.29-8.16 (m, 4H, Ar-H), $13.73\left(\mathrm{~s}, 1 \mathrm{H},-\mathrm{NH}\right.$, pyrazole); ${ }^{13} \mathrm{C}-\mathrm{NMR}\left(75 \mathrm{MHz}, \mathrm{DMSO}-d_{6}\right): \delta 14.7$, 26.5, 67, 105.8, 121, 127.4, 136, 144.1, 142, 145, 146.9, 161.6, 163.8. Mass (m/z): 343. Anal. (\%) for $\mathrm{C}_{15} \mathrm{H}_{13} \mathrm{~N}_{5} \mathrm{O}_{3} \mathrm{~S}$, Calcd. C, 52.47; H, 3.82; N, 20.41; S, 9.34. Found: C, 52.23; H, 3.60; N, 20.16; S, 9.19.

6-(4-Methylphenyl)-3,6-dimethyl-1,5,6,7-tetrahydro-8H-pyrazolo[3',4':4,5]thieno[2,3-d] pyrimidin-8-one (3g). Yield 44\%, mp. 304-306 ${ }^{\circ} \mathrm{C}$. IR (KBr): 3345, 3112, 2975, 1680, $681 \mathrm{~cm}^{-1}$; ${ }^{1} \mathrm{H}$ NMR (300 MHz, $\left.\mathrm{CDCl}_{3}\right): \delta 2.04\left(\mathrm{~s}, 3 \mathrm{H},-\mathrm{CH}_{3}\right.$, pyrimidine), $2.45\left(\mathrm{~s}, 3 \mathrm{H},-\mathrm{CH}_{3}\right), 2.76(\mathrm{~s}, 3 \mathrm{H}$, $\mathrm{CH}_{3}$, pyrazole), 7.03-7.09 (m, 4H, Ar-H), 8.05 (s, 1H, -NH, pyrimidine), 13.70 (s, 1H, -NH, pyrazole); ${ }^{13} \mathrm{C}-\mathrm{NMR}\left(75 \mathrm{MHz}, \mathrm{DMSO}-d_{6}\right): \delta 14.1,28.6,28.7,66.1,105.1,126.3,128.6,135$, 136, 136.2, 139, 142.1, 144.6, 166. Mass (m/z): 312. Anal. (\%) for $\mathrm{C}_{16} \mathrm{H}_{16} \mathrm{~N}_{4} \mathrm{OS}$, Calcd. C, 61.52; H, 5.16; N, 17.93; S, 10.26. Found: C, 61.29; H, 5.08; N, 17.56; S, 10.01.

\section{6-(4-Methoxylphenyl)-3,6-dimethyl-1,5,6,7-tetrahydro-8-pyrazolo[3',4':4,5]thieno[2,3-d]}

pyrimidin-8-one (3h). Yield 56\%, mp. 316-318 ${ }^{\circ} \mathrm{C}$. IR (KBr): 3340, 3110, 2973, 1682, 683, 1224, $1074 \mathrm{~cm}^{-1} .{ }^{1} \mathrm{H}$ NMR (300 $\left.\mathrm{MHz} \mathrm{CDCl}_{3}\right): \delta 2.04$ (s, 3H, - $\mathrm{CH}_{3}$, pyrimidine), 2.76 (s, 3H, $\mathrm{CH}_{3}$, pyrazole), 3.93 (s, 3H, $\left.-\mathrm{OCH}_{3}\right), 6.80-7.02$ (m, 4H, Ar-H), 8.02 (s, 1H, -NH, pyrimidine), $13.72\left(\mathrm{~s}, 1 \mathrm{H},-\mathrm{NH}\right.$, pyrazole); ${ }^{13} \mathrm{C}-\mathrm{NMR}\left(75 \mathrm{MHz}, \mathrm{DMSO}-d_{6}\right): \delta 14.6,28.6,55.9,66.9,105.8$, $113.9,127.6,134.1,135,141.3,142.6,144.1,158.3,162,163.9$. Mass (m/z): 328. Anal. (\%) for $\mathrm{C}_{16} \mathrm{H}_{16} \mathrm{~N}_{4} \mathrm{O}_{2} \mathrm{~S}$, Calcd. C, 58.52; H, 4.91; N, 17.10; S, 9.76. Found: C, 58.29; H, 4.56; N, 16.88; S, 9.56 . 


\section{Acknowledgements}

The authors are thankful to the Dept. Of Chemistry, Saurashtra University for providing laboratory facilities and CDRI Lucknow for providing ${ }^{1}$ HNMR and Mass spectral facilities. The authors are also thankful to Antimicrobial Acquisition and Co-ordination Facility (TAACF), U.S.A. for antitubercular activity.

\section{References}

1. Krikpatrick, W. E.; Okabe, T.; Hillyard, I. W.; Robins, R. K.; Dren, A. T.; Novinson, T. J. Med Chem. 1977, 20, 386.

2. Elagamy, A. A.; El-Taweel, F. M. A.; Amer, F. A.; Zoorob, H. H. Arch. Pharm. 1987, 246, 320.

3. (a) Kost, A. N.; Grandberg, I. I. In Advances in Heterocyclic Chemistry; A. R. Katritzky Ed. Academic Press: New York, 1996; pp 347. (b) Nihset, H. B. J. Chem. Soc. 1938, 12, 1568.

4. Raiziss, G. W.; Clemence, L. W.; Friefelder M. J. Am. Chem. Soc. 1941, 63, 2739.

5. Warner-Lambert Co., Jpn. Kokai Tokkyo Koho Jp. 61, 236, 778, 1986; Chem. Abstr. 1987, 20, $101967 \mathrm{~h}$.

6. Lindell, S. D.; Moloney, B. A.; Hewitt, B. D.; Earnhaw, C. G.; Philip,P. J.; Dancer, J. E. Bioorg. Med. Chem. Lett. 1999, 9, 1985.

7. Sanghvi, Y. S.; Larson, S. B.; Smee, D. F.; Revankar, G. R.; Robins, R. K. Nucleosides, Nucleotides. 1991, 10, 1417.

8. Bunnage, M. K.; Mathias, J. P.; Street S. D. A.; Wood A. PCT Int. Appl. WO, 14, 333, 487, 1999; Chem. Abstr. 1999, 131, $310644 \mathrm{f}$.

9. Bell, A. S.; Terrett, N. K. Eur. Pat. EP 526, 004, 1993; Chem. Abstr. 1993, 118, 254957c.

10. Doherty, P. C.; Place, V. A. Jr.; and Smith, W. L. PCT Int. Appl. 9921, 558 1999; Chem. Abstr. 1999, 130, 332908d.

11. (a) Szczech, G. M.; Furman, P.; Painter, G. R.; Barry, D. W.; Borroto-Esoda, K.; Grizzle, T. B.; Blum, M. R.; Sommadossi, J. P.; Endoh, R.; Niwa, T.; Yamamoto, M.; Moxham, C. Antimicrob. Agents Chemothe. 2000, 44, 123. (b) Giles, F. J.; Feldman, E. J.; Roboz, G. J.; Larson, R. A.; Mamus, S. W.; Cortes, J. E.; Verstovsek, S.; Federl, S.; Talpaz, M.; Beran, M.; Albitar, M., O’Brien, S. M.; Kantarjian, H. M. Leuk. Res. 2003, 27, 1091. (c) Giles, F. J. Expert Rev. Anticancer Ther. 2002, 2, 261. (d) Toyohara, J.; Hayashi, A.; Sato, M.; Gogami, A.; Tanaka, H.; Haraguchi, K.; Yoshimura, Y.; Kumamoto, H.; Yonekura, Y.; Fujibayashi, Y. Nucl. Med. Biol. 2003, 30, 687. (e) Mangner, T. J.; Klecker, R. W.; Anderson, L.; Shields, A. F. Nucl. Med. Biol. 2003, 30, 215. (f) Toyohara, J.; Fujibayashi, Y. Nucl. Med. Biol. 2003, 30, 681. (g) Dieterle, F.; Müller-Hagedon S.; Liebich, H. M.; Gauglitz, G. Artif. Intell. Med. 2003, 28, 265. 
12. (a) Rowley, D. C.; Hansen, M. S. T.; Rhodes, D.; Striffer, C. A.; Ni, H.; McCammon, J. A.; Bushman, F. D.; Fenical, W. Bioorg. Med. Chem. 2002, 10, 3619. (b) Arnaud,A.; Fontana, L.; Angulo, A. J.; Gil, Á.; López-Pedrosa, J. M. Clin. Nutr. 2003, 22, 391. (c) Gallant, J. E. J. Clin. Virol. 2002, 25, 317.

13. (a) Molina, P.; Aller, E.; Lorenzo, A.; Lopez-Cremades, P.; Rioja, I.; Ubeda, A.; Terencio, M. C.; Alcaraz M. J. J. Med. Chem. 2001, 44, 1011. (b) Kumar, A.; Sinha, S. P.; Chauhan, M. S. Bioorg. Med. Chem. Lett. 2002, 12, 667.

14. (a) Bher, J. B.; Gourlain, T.; Helimi, A.; Guillerm. G. Bioorg. Med. Chem. Lett. 2003, 13, 1713. (b) De Koning, H. P.; Al-Salabi, M. I.; Cohen, A. M.; Coombs, G. H.; Wastling, J. M. Int. J. Paras. 2003, 821. (c) Behr, J. B.; Gourlain, T.; Helimi, A.; Guillerm, G. Bioorg. Med. Chem. Lett. 2003, 13, 1713. (d) De Koning, H. P.; Al-Salabi, M. I.; Cohen, A. M.; Coombs, G. J.; Wastling, M. Int. J. Paras. 2003, 33, 821.

15. Nakum, S. D.; Pavagadhi, T. H.; Shah, V. H. Oriental J. Chem. 2002, 18, 525: Chem. Abstr. 2002, 137, 279122.

16. Nakum, S. D.; Sikotra, K. H.; Shah, V. H. Ind. J. Het. Chem. 2002, 12, 37; Chem. Abstr. 2002, 138, 187729.

17. Parikh, A. R.; Gaur, V. B.; Shah, V. H. J. Inst. Chem. 1991, 63, 219; Chem. Abstr. 1993, 119, 49324y.

18. Parikh, A. R.; Chauhan, N. A.; Shah, V. H. J. Ind. Chem. 1987, 64, 678; Chem. Abstr. 1988, $109,210992 \mathrm{v}$.

19. Gewald, K. Chem. Heterocycl. Compd. (Engl.Transl.) 1976, 12, 1077; Chem. Abstr. 1968, 68, 49383v.

20. Taylor, E. C.; Berger, G.; J. Org. Chem. 1967, 32, 2376; Chem. Abstr. 1967, 67, 64348k.

21. Arya, V. P.; Shenoy, S. J. Ind. J. Chem. 1972, 10, 815; Chem. Abstr. 1973, 78, 84312z.

22. Rosoasky, A.; Chen, K. K. N.; Lin M. J. Med. Chem. 1973, 16, 191; Chem. Abstr. 1973, 79, 49031x.

23. Sharanina, L. G.; Barana, S. N. Chem. Het. Compd. (Engl. Transl) 1974, 10, 171; Chem. Abstr. 1974, 80, 133379d.

24. Swelam, S. A.; Abdel Salam M. E. A., Zaki, O. L. Ind. J. Het. Chem. 1999, 257.

25. Franzblau, S. G.; Witzig, R. S.; McLaughlin, J. C.; Torres, P.; Madico, G.; Hernandez, A.; Degnan, M. T.; Cook, M. B.; Quenzer, V. K.; Freguson R. M.; Gilman, R. H. J. Clin. Microbiol. 1998, 36, 362.

26. Collins, L. A.; Franzblau, S. G. Antimicrob. Agents Chemother. 1997, 41, 1004. 